\title{
Health Policy and Health Communication: A Case of China's Zero COVID Policy
}

Opinion
Volume 3 Issue 2- 2022
Author Details
Qian Liu*
School of Journalism and Communication, Jinan University, China
*Corresponding author
Qian Liu, School of Journalism and Communication, Jinan University, China
Article History
Received: February 11, $2022 \quad$ Accepted: February 14, $2022 \quad$ Published: February 18, 2022

\begin{abstract}
This article attempts to elaborate on China's zero COVID policy, especially focus on the health system policy, health communication and traffic restriction. Effective health policies and proper health communication would support the building of a system to copy with the virus.

Keywords: COVID-19; China; Zero COVID Policy; Health Communication; Health Policy; Omicron; Health System Policy; Health Communication Policy; Traffic Restriction
\end{abstract}

\section{Introduction}

In December 2019, early COVID-19 cases were reported from Wuhan city, in Hubei, China. Wuhan went through a lockdown as a health policy on January $23^{\text {rd }} 2020$. Scientists claim COVID-19 will continue but the end of the pandemic is near [1], and we still need to deal with health policies and health communication about COVID. With lockdown of cities like Wuhan and Xi' an, mass COVID testing China's stringent zero COVID strategy is working but still facing challenges especially with asymptomatic infections for Omicron and millions of traveling people for Winter Olympics [2] and Chinese New Year in 2022.

Health policies ranging from the strictest ones such as city lockdown to the milder ones such as wearing of masks, keeping social distance have been adopted by different governments around the world and have saved the lives of many. Health policy as an important public health issue has been studied as an important academic topic in COVID-19 researches and could help effective decision-making process [3]. According to specific situation government made different choices to prevent the spread of the virus the different policies have caused not only great public attention but also discussions. Researchers even simulated different scenarios with model to see outcome for different sets of policies [4]. For China in terms of vaccines it has been reported a total of 3,004.681 million doses of the new coronavirus vaccine taken by February 52022 [5], which covered over 75\% of the population [6]. CoronaVac and Sinopharm vaccines are the vaccine widely used in china. With the inactivated-virus technology researchers found it to be with some protection against severe disease with Omicron but not with a lot of prevention for infections [7].

Health communication including mass media and new media played a crucial role in educating citizens about epidemic policies, health information in various forms such as news reports, documentaries, movies, TV series, social media [8]. For example, "Wuhan War Epidemic" is a documentary published by CGTN (China Global Television Network) about the whole process of Wuhan's lockdown as health measure communicating with global citizens on YouTube platform. Digital contact tracing apps were also used in some countries to communicate and benefit citizens as well, researchers evaluated the effectiveness and usefulness of COVID Safe, an Australia's tracing app for COVID-19, and found it useful [9]. The Chinese government has made a series of health policies and focus on the health communication in the fight against the epidemic. The following is an introduction to the standardized management of health system policy, health communication and traffic restriction.

\section{Health System Policy}

In the "Technical Guidelines for the Prevention and Control of Novel Coronavirus Infection in Medical Institutions (Third Edition)", 
technical guidelines are listed in three parts by government: general requirements, prevention and control strategies and basic requirements [10].

The general requirements and main purpose are to increase the attention and standardization of the prevention and control of new COVID-19 infection in medical system.

For prevention and control strategies it proposes the followings: (1) On the basis of "early detection, early reporting, early isolation, and early treatment" internal and external prevention should be done well (2) Prevention between doctors and patients (3) Prevention between people and objects (4) Integration of "three defenses": people, technical, device.

Among the basic requirements it suggests the followings: (1) Improve the prevention and control system, work flow and emergency plan (2) Focus on source control and strictly prevent the risk of infection from being imported into medical system (3) Carry out training for all staff to improve the awareness and level of infection prevention and control (4) Strengthen prevention (5) Strengthen personnel control and reduce crowd gatherings (6) Care for medical staff and implement health monitoring (7) Carry out risk assessment (8) Strengthen health education.

In addition, specific technical requirements are also made for key departments such as emergency departments. ${ }^{1}$

\section{Health Communication and Traffic Restriction}

The multi-departmental collaborative management is the core of the anti-epidemic policy. The health communication and traffic restriction related policies are the focus during the Spring Festival and the 2022 Winter Olympics time in China which needs the multi-departmental collaborative management.

China's Lunar New Year is a traditional festival with the largest flow of people moving around the country. The Winter Olympics are also held during this period in 2022. Winter is a season for virus to be more active as well. So, the risk of epidemic transmission has increased which has brought great challenges to prevention and control. The government has formulated the "2022 Spring Festival Plan for Comprehensive Transportation Epidemic Prevention, Control and Service Guarantee" [11] from January 17 to February 252022 to ensure the joint efforts of multiple departments. The following points have been made:

a) Scientifically prepare for the epidemic prevention and control.

b) Make every effort to ensure the safe and orderly operation of transportation.

c) Strengthen the guarantee for passengers' travel services.

d) Strengthen the road network and ensure smooth transportation of key materials.

e) Set clear job responsibilities, in aspects of: (1) local area's responsibility (2) Improve the working mechanism (3) Strengthen

${ }^{1}$ Some policies are omitted limited to space requirements, please check the official website for detailed and accurate information. the dynamic monitoring (4) Strengthen information reporting and sharing and (5) Strengthen the communication and the guidance of public opinion.

\section{Conclusion}

Health policy and health communication are of vital importance for the fighting against COVID-19. With China's zero COVID policy introduced by the central government policies such as health system policy, health communication policy and traffic restriction have been carried out involving lockdown, mass testing, vaccine, social distance, travel bans and others. Proper health policy and health communication implemented could significantly improve the public health. In the future zero COVID policy still are facing a lot of challenges in China. Omicron is spreads so quickly [12] specific vaccines are urgently needed suggested by scientists [13]. Besides effects of scientists, effective health policies and proper health communication would together collaborate and support the building of a system to copy with the virus.

\section{References}

1. Murray CJL (2022) COVID-19 will continue but the end of the pandemic is near. The Lancet 399(10323): 417-419.

2. Mallapaty S (2022) China's zero-COVID strategy: what happens next? Nature 602(7895): 15-16.

3. Yu S, Qing Q, Zhang C, Shehzad A, Oatley G, et al. (2021) Data-Driven Decision-Making in COVID-19 Response: A Survey. IEEE Trans Comput Soc Syst 8(4): 1016-1029.

4. Kuzdeuov A, Baimukashev D, Karabay A, Ibragimov B, Mirzakhmetov A, et al. (2020) A Network-Based Stochastic Epidemic Simulator: Controlling COVID-19 With Region-Specific Policies. IEEE J Biomed Health Inform 24(10): 2743-2754.

5. (2022) COVID-19 vaccination status (as of February 5).

6. Silver A (2021) Covid-19: Why China is sticking to "zero tolerance" public health measures. BMJ 375: n2756

7. Normile D (2021) 'Zero COVID' is getting harder-but China is sticking with it. Science 374(6570): 924.

8. Li L, Zhang Q, Wang X, Zhang J, Wang T, et al. (2020) Characterizing the Propagation of Situational Information in Social Media During COVID-19 Epidemic: A Case Study on Weibo. IEEE Trans Comput Soc Syst 7(2): 556-562.

9. Vogt F, Haire B, Selvey L, Katelaris AL, Kaldor J (2022) Effectiveness evaluation of digital contact tracing for COVID-19 in New South Wales, Australia. The Lancet Public Health.

10. (2021) Notice on Issuing Technical Guidelines for Prevention and Control of Novel Coronavirus Infection in Medical Institutions.

11. (2022) Notice on Printing and Distributing the "Overall Work Plan for the Prevention and Control of Epidemic Prevention and Transportation Service Guarantee for the Spring Festival Transportation of Comprehensive Transportation in 2022".

12. Ahmad Z, Zubair I, Zuber N Is Omicron Less Severe than Delta? 2.

13. Carreño JM, Alshammary H, Tcheou J, Singh G, Raskin A, et al. (2021) Activity of convalescent and vaccine serum against SARS-CoV-2 Omicron. Nature p. 1-8. 